\title{
LE NUCLÉAIRE FACE À L'OPINION PUBLIQ̣UE
}

\section{Analyses, stratégies de communication, actions}

$D$

ans la sphère des relations multiformes qui s'établissent entre les différents acteurs de la vie sociale, l'énergie nucléaire engendre un problème de communication d'une rare complexité.

Le sujet en lui-même met en jeu des notions scientifiques et techniques difficilement réductibles à la simplification. Et pourtant, nous dit-on, pour communiquer «il faut faire simple...", premier obstacle ou défi à relever dans le cadre d'un dialogue avec le grand public...

Les acteurs nucléaires sont diversifiés : organismes de recherche, industriels, exploitants, autorités réglementaires, sociétés savantes, etc. Leurs politiques d'information n'obéissent pas aux mêmes impératifs, leurs messages sont différents, les publics destinataires ne sont pas identiques, même s'ils se recoupent parfois : deuxième difficulté à prendre en compte pour produire à destination de l'opinion un "message» nucléaire perçu comme cohérent...

Ce public lui-même est caractérisé d'abord par sa diversité : âge, sexe, niveau d'instruction; attitudes d'approbation, d'hostilité, d'indifférence; lieux d'habitation plus ou moins proches d'installations nucléaires, etc... troisième obstacle à surmonter pour conduire un dialogue adapté à la diversité des interlocuteurs...

A ces difficultés - qui ne sont que quelques-unes parmi d'autres - s'ajoute l'inévitable distorsion que subit n'importe quel discours dès lors que l'on ne se trouve plus en situation de communication directe mais en situation de communication médiatisée, ce qui est le cas de figure le plus fréquent. Les "producteurs" d'information nucléaire savent bien que lorsqu'ils disent "A» le message reçu par le public via le médiateur ne sera pas forcément "A" mais le plus souvent " $A$ "», voire " $B » \ldots$ Autrement dit, produire une information ne signifie pas automatiquement la communiquer : autre obstacle sur la voie d'un dialogue clair à l'abri des malentendus...

Qu'en conclure, sinon que les personnes chargées d'étudier ou de pratiquer la communication nucléaire rencontrent nécessairement dans leur lot quotidien "ces repentirs, ces doutes, ces contraintes» dont parle le poètè... et que leur démarche ne peut se concevoir que dans la rigueur et un professionnalisme particulièrement affirmés. C'est de tout cela que témoignent avec une franchise souvent décapante les articles du présent dossier. Nous remercions vivement les auteurs d'avoir bien voulu nous livrer cette part de réflexion et d'expérience comme un regard posé de l'intérieur sur la complexité de leur métier.

Nos remerciements vont également à Mme Véronique Lafrance pour la part qu'elle a prise dans l'élaboration de ce dossier.

Francis SORIN 